ISSN 0103-5150

Fisioter. Mov., Curitiba, v. 25, n. 2, p. 419-431, abr./jun. 2012 Licenciado sob uma Licença Creative Commons

\title{
Subsídios reunidos junto à equipe de saúde para a inserção da fisioterapia na Estratégia Saúde da Família
}

\author{
Information gathered with health team to support the \\ insertion of physical therapy in Family Health Strategy
}

\section{Cristina Pellegrino Baena ${ }^{[a]}$, Maria Cristina Flores Soares ${ }^{[b]}$}

[a] Mestre em Ciências da Saúde pela Universidade Federal do Rio Grande (FURG), Rio Grande, RS - Brasil, e-mail: crispel13@hotmail.com

[b] Docente do Programa de Pós-Graduação em Ciências da Saúde (PPGCiSau) e do Instituto de Ciências Biológicas (ICB) da Universidade Federal do Rio Grande (FURG), Rio Grande, RS - Brasil, e-mail: mcflores01@gmail.com

\section{Resumo}

Esta pesquisa visou obter subsídios para a inserção do fisioterapeuta na Estratégia Saúde da Família em Rio Grande/RS. Estudo quantitativo, delineamento transversal com 196 trabalhadores. Utilizou-se instrumento semiestruturado com os temas: princípios norteadores do SUS/ESF, identificação de situações com potencialidade para a atuação fisioterápica, percepção sobre preparo da equipe para enfrentá-las e infraestrutura das unidades. Foi realizada a distribuição de frequências das variáveis, a comparação das médias (ANOVA) e o teste de Tukey. As médias das notas atribuídas aos princípios integralidade, equidade e universalidade diferiram entre si $(p=0,00)$, sendo $6,3 \pm 2,4,7,0 \pm 2,4$ e 7,8 $\pm 2,2$, respectivamente. As situações mais prevalentes foram: hipertensão (90,8\%), diabete $(89,8 \%)$, doenças osteomusculares $(88,2 \%)$, DPOC $(84,7 \%)$, pessoas acamadas (83,7\%), dificuldade para caminhar (83,7\%) e deficientes físicos (82,7\%). Quanto ao preparo da equipe, a maioria dos trabalhadores afirmou se sentir despreparado para: atraso do desenvolvimento neuropsicomotor (51,7\%), treino de marcha (51,8\%), atenção a deficientes físicos $(68,1 \%)$ e amputados (57,0\%). Na avaliação da infraestrutura das unidades, a maioria acusou bom estado do prédio e unidades, com salas para atendimento especializado $(31,6 \%)$. Os dados evidenciam as possibilidades de ação do fisioterapeuta na ESF, visando aumentar a resolubilidade da mesma.

Palavras-chave: Fisioterapia. Promoção de saúde. Saúde da família. 
Abstract

This study aimed to subsidize Physical Therapists (PT) in the Family Health Strategy (FHS) in Rio Grande, Rio Grande do Sul. It is a quantitative study with cross-cut delimitation with 196 workers in the FHS. A semistructured instrument approached the subjects: guideline criteria of FHS, identification of existing situations for the physiotherapy performance, staff's perception about the readiness to face those situations and the infrasctructure. A frequency distribution of the variables, averages comparison (ANOVA) and the Tuckey test were made. The means of the grades assigned to integrality, equity and universality differed among each other $(p=0.00): 6.3 \pm 2.4,7,0 \pm 2.4$ and $7.8 \pm 2.2$, respectively. The most prevailing pathologies identified were: hypertension (90.8\%), diabetes (89.8\%), musculoskeletal diseases (88.2\%), chronic obstructive pulmonary disease (84.7\%), bedridden (83.7\%), walking difficulties (83.7\%) and physically handicapped (82.7\%). It was noticed that in five situations, most of the workers stated as unprepared to face them: developmental delay (51.7\%), gait training (51.8\%), disabled (68.1\%) and amputees (57.0\%). In the evaluation of the infrastructure of the units, most of the workers reported the building as in good and specialized treatment room (31.6\%). Our data aids the possibility of the PT placement in the ESF city staff, increasing its solvability.

Keywords: Physiotherapy. Health promotion. Family health.

\section{Introdução}

O acesso democratizado e a gestão descentralizada são passos importantes quando se considera o antigo panorama de exclusão e iniquidades em relação à saúde. Apesar de ser palco de grandes contradições, o Sistema Único de Saúde (SUS) é o sistema de saúde do povo brasileiro legitimado em Constituição Federal, sendo considerado como o maior projeto público de inclusão social brasileiro (1).

Por estruturar-se nos princípios de universalidade, equidade e integralidade das ações de prevenção, tratamento e reabilitação de doenças, a promoção de saúde incorpora-se como peça chave para a eficácia do sistema (2).

Nessa perspectiva, o Ministério da Saúde assumiu como estratégia setorial e reorientação do modelo assistencial brasileiro, a partir de 1994, o Programa de Saúde da Família (PSF). Baseado no modelo de atenção à saúde de países como Cuba, Canadá, Suécia e Reino Unido, sua concepção levou em consideração experiências nacionais como as de Sobral, CE, e Londrina, PR, e experiências em nível estadual como a da Secretaria Estadual da Saúde do Estado do Rio Grande do Sul. A partir de 2006, na chamada Reforma Estratégica do SUS e no Pacto em Defesa da Vida, esse programa passou a ser denominado Estratégia Saúde da Família - ESF (3).

A abordagem do indivíduo em seu meio familiar proporcionou ao sistema uma maior abrangência na atenção à saúde, a qual passou a ser encarada na prática profissional como fenômeno socialmente determinado. Com isso, a interdisciplinaridade, indispensável para o aumento da resolubilidade na atenção básica, passa a ser muito valorizada. Nessa perspectiva, a equipe atuante no início do programa, constituída minimamente por agentes comunitários de saúde (ACSs), enfermeiros e médicos, recebeu a inserção regular de odontólogos e técnicos em odontologia desde 2000 (4).

No entanto, maior abrangência e resolubilidade da atenção básica à saúde dar-se-ão na medida em que outras áreas da saúde ou do conhecimento de uma forma geral possam (co)atuar em favor da integralidade em saúde, no efetivo exercício da interdisciplinaridade (5). Assim, a discussão sobre a reformulação da equipe da ESF torna-se parte do processo de adequação constante do SUS em relação às demandas da população.

Atualmente, algumas equipes em diferentes regiões do país são integradas por outros profissionais, como nutricionistas, psicólogos, fisioterapeutas e educadores físicos, refletindo um fenômeno de adequação às necessidades locais $(6,7)$. Essas inserções isoladas resultam, possivelmente, de uma percepção da própria equipe que está em contato com a comunidade e dependem da vontade política dos gestores municipais.

Os relatos de inclusão de outros profissionais à ESF causam especial reflexão entre os fisioterapeutas 
(8).É fato que, historicamente, a Fisioterapia tem assumido seu papel na reabilitação, mas durante esses 40 anos desde a sua regulamentação, a profissão tem passado por um processo de ampliação do campo de atuação em função do desenvolvimento tecnológico e de novas demandas da clientela (população) em nível individual e coletivo $(9,10)$.

O fisioterapeuta é, hoje, um profissional que deve estar preparado para atuar em todos os níveis de atenção à saúde, incluindo a prevenção, a promoção, o desenvolvimento, o tratamento e a recuperação da saúde em indivíduos, grupos de pessoas ou comunidades (9).

Essa perspectiva é resumida por Silva e DaRos (4): "Mais do que recuperar e curar pessoas, é preciso criar condições necessárias para que a saúde se desenvolva", deixando clara a preocupação com a capacitação para a promoção de saúde. 0 questionamento da necessidade da competência específica do fisioterapeuta enquanto promotor de saúde na rede de atenção básica vem da maturidade social conquistada pela profissão (11). Esse aspecto pode ser constatado em relatos da atuação desse profissional na ESF que têm sido descritos $(12,4,13)$.

Formalmente, a inclusão de fisioterapeutas nos Núcleos de Apoio à Saúde da Família - NASF tornou-se possível recentemente, como resultado da discussão ainda em processo no Ministério da Saúde sobre o tema da integralidade, definida como "o esforço da equipe de saúde para atender da melhor forma possível as necessidades da saúde, devendo estas serem captadas em sua expressão individual" (3).

A partir das vivências do cotidiano profissional, das inquietações e reflexões sobre as novas demandas da saúde da população e da falta de subsídios na literatura para uma atuação eficaz na perspectiva da ESF junto à realidade local que, atualmente, não conta com a Fisioterapia em nível de atenção primária, surgiu o tema desta pesquisa. Tendo como perspectiva a promoção de saúde, no contexto da ESF postula-se que o fisioterapeuta pode vir a compor a equipe ESF, contribuindo assim para o aumento da resolubilidade da atenção básica à saúde e para a efetivação da integralidade no SUS.

Este estudo tem como objetivo buscar subsídios, com base na prática diária e na realidade local vivenciada pelos profissionais que constituem as equipes de saúde do município do Rio Grande, para estimular a inserção/atuação do fisioterapeuta nas equipes da ESF.

\section{Materiais e métodos}

Trata-se de um estudo quantitativo, com delineamento transversal, realizado no município do Rio Grande/RS. Fizeram parte da população do estudo os trabalhadores da ESF. Dos 206 trabalhadores em atividade, $196(95,1 \%)$ foram entrevistados. Os motivos verificados para não participação na pesquisa de alguns trabalhadores foram: recusas (7) e férias em duas visitas consecutivas à unidade no final do período da coleta de dados (3).

Foi utilizado um instrumento misto semiestruturado com questões de escolha simples ${ }^{(*)}$, múltipla escolha ${ }^{(* *)}$ ou com atribuição de nota aos diversos itens apresentados, de acordo com a realidade vivenciada pelo entrevistado ( 1 para mínimo e 10 para máximo $)^{(* * *)}$. A coleta foi realizada entre junho e novembro de 2009, por dois entrevistadores treinados.

As variáveis investigadas foram: idade; sexo; tempo de atuação na unidade; localização da unidade (zona urbana ou rural); características da formação dos membros da equipe: graduados em saúde (Grupo GS: médicos e enfermeiras) ou não graduados em saúde (Grupo NGS - ACSs, técnicos e auxiliares de enfermagem) e história de especialização em Saúde da Família; princípios norteadores do SUS e ESF aplicados na prática diária (universalidade, equidade e integralidade) ${ }^{(* * *)}$; situações com potencialidade para atuação da Fisioterapia na atenção básica - comunidade $^{(*)}$, conforme identificadas por diferentes autores, e a percepção/opinião do entrevistado sobre o nível de preparação da equipe (com sua composição atual) para atender a essas situações ${ }^{(*)}(14,15,4$, 16). Ressalta-se que, nesta última questão, o sujeito deveria escolher uma das seguintes opções: equipe preparada, parcialmente preparada ou despreparada. Foram, ainda, investigados a infraestrutura existente nas unidades de saúde ${ }^{(* *)}$ e a opinião do entrevistado sobre a formação em Fisioterapia e sua autonomia ${ }^{(*)}$, níveis de atuação ${ }^{(*)}$ e contato prévio com a atuação do fisioterapeuta ${ }^{(* *)}$.

Para garantir o anonimato dos sujeitos da pesquisa as unidades foram renumeradas evitando coincidência entre o número da unidade designado pela Secretaria de Saúde do município e o adotado na pesquisa. Foi solicitada adesão dos trabalhadores por meio da assinatura, em duas vias, do Termo de Consentimento Livre e Esclarecido. 0 projeto foi analisado e aprovado pelo Núcleo de Pesquisa da Secretaria de Saúde Municipal (NEPES) e pelo 
Conselho de Ética em Pesquisa em Saúde (CEPAS), da FURG, sob parecer n. 48/2009.

0 controle de qualidade se caracterizou pela supervisão contínua do trabalho de campo, revisão diária dos questionários e dupla digitação seguida pela avaliação dos dados e análise de consistência dos mesmos.

Os dados foram bidigitados em uma planilha EpiInfo 6 e a análise estatística realizada com o auxílio do pacote estatístico Stata versão 10. Inicialmente, foi realizada uma análise descritiva com distribuição das frequências das variáveis, seguida de um estudo comparativo entre os diferentes grupos utilizando-se o teste qui-quadrado.

A análise das questões com atribuição de notas de 1 a 10 foi realizada de duas formas. Primeiramente, foram calculadas as médias \pm desvio padrão da média e comparação das mesmas por análise de variância, seguida pelo teste de Tukey. Essas médias foram também categorizadas em $\geq 5$ ou $<5$ e comparadas em relação à localização das unidades de saúde (zona rural ou urbana), e graduação ou não dos profissionais na área da saúde (grupo GS ou GSN). Foram considerados significantes os valores em que $\mathrm{p}<0,05$.

\section{Resultados e discussão}

\section{Caracterização da amostra}

Fizeram parte do estudo 196 trabalhadores em atividade da ESF municipal de acordo com a Tabela 1. Quando dicotomizados quanto à formação, obteve-se um grupo constituído por 50 profissionais com graduação na área da saúde (grupo GS formado por médicos e enfermeiros) e outro formado por ACSs, técnicos e auxiliares de enfermagem (Grupo GNS; $\mathrm{n}$ =146). Quando o grupo de enfermeiros e médicos foi categorizado quanto à realização de especialização em Saúde da Família, verificou-se que 67,4\% possuíam esta especialização.

Princípios que norteiam as práticas diárias das equipes de saúde

No que se refere à aplicação na prática diária, dos princípios fundamentais e norteadores do SUS e ESF, a média das notas atribuídas pelos trabalhadores constam na Tabela 2.
Tabela 1 - Dados sociodemográficos dos trabalhadores da ESF, Rio Grande, RS, 2009

\begin{tabular}{lc}
\hline \multicolumn{2}{c}{ Caracterização da amostra } \\
\hline Sexo & $86,2 \%$ Feminino \\
Idade média & $36,2 \pm$ anos \\
Tempo médio de atuação & $34,1 \pm 30,7$ meses \\
Zona urbana & $67,3 \%$ \\
Grupo GS & 50 indivíduos \\
Especialidade SF & $67,4 \%$ dos GS \\
Grupo NGS & 146 indivíduos \\
\hline
\end{tabular}

Fonte: Dados da pesquisa.

Tabela 2 - Média das notas atribuídas pelos trabalhadores à prática dos princípios norteadores da ESF, Rio Grande, RS, 2009

\begin{tabular}{lc}
$\begin{array}{l}\text { Princípios que norteaim as práticas } \\
\text { diárias das equipes de saúde }\end{array}$ & $\begin{array}{c}\text { Nota média } \\
(\mathbf{p}>\mathbf{0 , 0 0})\end{array}$ \\
\hline Universalidade & $7,8 \pm 2,2$ \\
Equidade & $7,0 \pm 2,4$ \\
Integralidade & $6,3 \pm 2,4$
\end{tabular}

Fonte: Dados da pesquisa.

Especificamente quanto à integralidade, quando as médias foram categorizadas ( $\geq 5$ ou $<5$ ) e comparadas em relação à localização das unidades de saúde, verificou-se que uma porcentagem significativamente maior $(p=0,04)$ de trabalhadores da zona rural $(54,3 \%)$ atribuiu notas inferiores a 5 quando comparada aos da zona urbana $(39,2 \%)$.

Não houve diferença significativa entre as médias das notas dadas pelos profissionais (GS) com ou sem graduação na área da saúde (NGS). Resultado semelhante foi verificado quando comparados os dois grupos de profissionais com ou sem especialização em Saúde da Família.

Alguns autores têm afirmado que o PSF representa um esforço bem-sucedido de equidade, em função de seu melhor desempenho em regiões de maior iniquidade (17).

0 item universalidade recebeu a maior nota média entre os entrevistados como o princípio melhor consolidado na prática diária desses trabalhadores. Entretanto, a efetivação da universalidade tem sido avaliada como parcial em razão da falta de cobertura 
em algumas áreas (10). Esse aspecto se confirma no município em estudo, no qual a cobertura da ESF ainda não atinge $40 \%$ da população (18).

A menor nota média atribuída à integralidade indica que este é um campo de debate não só por representar uma renúncia ao reducionismo na atenção à saúde, mas também pela discussão da interdisciplinaridade inerente ao tema.

Em uma reflexão sobre a integralidade e a composição das equipes da ESF, Loch-Neckel et al. (19) relatam que é a partir do trabalho interdisciplinar que se alcança uma abordagem integral sobre os fenômenos que interferem na saúde da população, atingindo maior eficácia dos programas e serviços oferecidos à população.

Para alguns componentes do Ministério da Saúde (20), a implementação da integralidade representa o maior desafio da saúde no Brasil. Sua análise como eixo prioritário de políticas de saúde pode se dar em três dimensões: da organização dos serviços de saúde, da adequação da formação e das práticas dos trabalhadores nos serviços de saúde e da formulação de políticas com a participação dos atores envolvidos na produção do cuidado. Assim, a integralidade é definida legal e institucionalmente como um conjunto articulado de ações e serviços de saúde, preventivos e curativos, individuais e coletivos, em cada caso, nos níveis de complexidade do sistema. Para Mattos (21), a integralidade representa uma boa prática para todo profissional de saúde, qualquer que seja seu local de atuação.

A identificação do princípio da integralidade como o menos efetivo também ressalta a necessidade de readequação das equipes diante da realidade local. Além disso, o fato de uma maior porcentagem de trabalhadores das equipes de saúde das unidades da zona rural ter atribuído uma nota média inferior a esse princípio sugere que a aplicação limitada na prática diária da integralidade enquanto instrumento da promoção, prevenção e recuperação da saúde poderia representar um impacto negativo ainda mais importante sobre a resolubilidade da ESF na zona rural.

Uma possibilidade de exercício mais efetivo da integralidade pela ESF apresenta-se desde 2008, quando o MS lançou a portaria GM 154, que criou os Núcleos de Apoio à Saúde da Família (NASF), que pretendem ampliar a abrangência das ações de atenção básica por meio da participação interdisciplinar. Essa Portaria prevê a inserção de fisioterapeutas aos NASFs, assim como médicos acupunturistas, assistentes sociais, profissionais de educação física, farmacêuticos, fonoaudiólogos, psicólogos e terapeutas ocupacionais. Essas equipes devem ser compostas por três ou cinco profissionais, dependendo da população a ser atendida e de acordo com as necessidades locais.

Identificação de situações com potencialidade para atuação da fisioterapia na comunidade

Quatorze situações em que a ação da fisioterapia na atenção básica à saúde tem sido descrita e indicada como necessária foram apresentadas aos trabalhadores para identificação das mesmas em sua prática diária (14-16, 22). Todas as situações foram identificadas por mais de $60 \%$ dos entrevistados, conforme Tabela 3.

A atenção em fisioterapia ainda na unidade básica e/ou no próprio domicílio poderia contribuir para a resolubilidade ou para uma redução do agravamento de situações que comumente são beneficiadas pela ação desse profissional $(8,23)$.

Outro aspecto importante refere-se à possibilidade de redução de internações hospitalares, que poderia ser vislumbrada com a atuação da fisioterapia na comunidade (unidade básica e/ou domicílio). É importante ressaltar que a análise da distribuição percentual das internações por grupo de causas - SIH/SUS, 2007, aponta para uma grande prevalência de hospitalizações no município por doenças crônicas, muitas delas referenciadas pelos trabalhadores de saúde neste estudo (18).

A Tabela 3 mostra que quando os sujeitos da pesquisa foram categorizados pela graduação em saúde (GS) ou não (NGS), verificou-se que médicos e enfermeiros identificaram uma porcentagem significativamente maior das seguintes situações: gestantes, crianças com atraso no desenvolvimento neuropsicomotor, pessoas acamadas, pessoas com doenças osteomusculares, pessoas com dificuldade de deambulação, adultos com DPOC, crianças com doenças pulmonares, deficientes físicos, cuidadores de pessoas acamadas e amputados.

Há uma menor identificação de situações que requerem atenção integral à saúde por parte dos ACSs. A falta de um treinamento formal e o foco nas visitas domiciliares dos ACSs, descritos por Pedrosa e Teles (24), poderiam auxiliar na explicação desses resultados. Contrariamente, os profissionais com graduação em saúde (grupo GS) têm seu foco principal 
Tabela 3 - Distribuição de frequência das situações com potencialidade para atuação da fisioterapia na atenção básica identificadas pela totalidade dos trabalhadores que constituem as equipes da ESF e pelos grupos de graduados (GS) e não graduados em saúde (NGS), Rio Grande, RS, 2009

\begin{tabular}{|c|c|c|c|c|c|c|c|}
\hline & \multicolumn{2}{|c|}{ Total } & \multicolumn{2}{|c|}{ GS } & \multicolumn{2}{|c|}{ NGS } & \multirow[b]{2}{*}{$\mathbf{p}$} \\
\hline & $\mathbf{n}$ & $\%$ & $\mathrm{n}$ & $\%$ & n & $\%$ & \\
\hline Preparo de gestantes para o parto e aleitamento materno & 148 & 75,5 & 00 & 92,0 & 00 & 69,9 & $0,02^{*}$ \\
\hline Atividades físicas e orientações quanto ao autocuidado para diabéticos & 176 & 89,8 & 47 & 94,0 & 129 & 88,4 & 0,25 \\
\hline $\begin{array}{l}\text { Atividades físicas e orientações para melhorar a qualidade } \\
\text { de vida de hipertensos }\end{array}$ & 178 & 90,8 & 48 & 96,0 & 130 & 89,0 & 0,14 \\
\hline Orientação e estimulação a crianças com atraso do DNPM & 127 & 64,8 & 40 & 80,0 & 87 & 59,6 & $0,00^{*}$ \\
\hline $\begin{array}{l}\text { Assistência e orientação sobre prevenção de escaras, posicionamento, } \\
\text { alongamento e fortalecimento em pessoas acamadas }\end{array}$ & 164 & 83,7 & 47 & 94,0 & 117 & 80,1 & $0,02^{*}$ \\
\hline $\begin{array}{l}\text { Assistência e orientação sobre analgesia, posicionamento, } \\
\text { alongamento, fortalecimento e retorno às atividades funcionais em } \\
\text { pessoas com doenças osteomusculares }\end{array}$ & 172 & 88,2 & 49 & 98,0 & 123 & 84,8 & $0,01^{*}$ \\
\hline $\begin{array}{l}\text { Orientação sobre prevenção de agravos crônico-degenerativos e } \\
\text { manutenção da saúde }\end{array}$ & 145 & 74,0 & 43 & 86,0 & 102 & 69,8 & $0,03^{*}$ \\
\hline Treino de marcha para pessoas com dificuldade para deambular & 164 & 83,7 & 46 & 92,0 & 118 & 80,8 & 0,04 * \\
\hline Higienização brônquica e orientação respiratória para adultos com DPOC & 166 & 84,7 & 47 & 94,0 & 119 & 81,5 & $0,03^{*}$ \\
\hline $\begin{array}{l}\text { Higienização brônquica e orientação respiratória para crianças com } \\
\text { doenças pulmonares }\end{array}$ & 137 & 69,9 & 41 & 82,0 & 96 & 65,8 & $0,03^{*}$ \\
\hline Assistência especializada e/ou reabilitação para deficientes físicos & 162 & 82,7 & 47 & 94,4 & 119 & 78,8 & $0,01^{*}$ \\
\hline Orientação a idosos quanto a prevenção de quedas & 149 & 76,4 & 42 & 84 & 107 & 73,8 & 0,14 \\
\hline $\begin{array}{l}\text { Orientação quanto a postura e autocuidado para cuidadores } \\
\text { de pessoas acamadas }\end{array}$ & 155 & 79,1 & 46 & 92,0 & 109 & 74,7 & $0,00^{*}$ \\
\hline $\begin{array}{l}\text { Assistência especializada e/ou reabilitação para amputados } \\
\text { com ou sem próteses }\end{array}$ & 124 & 63,3 & 42 & 84 & 82 & 56,2 & $0,00^{*}$ \\
\hline
\end{tabular}

Fonte: Dados da pesquisa.

Legenda: $\mathrm{n}=$ número de trabalhadores; * $=$ diferença significativa entre os grupos GS e NGS.

de atuação nas consultas dentro da unidade, atendendo a uma demanda concentrada de agravos acima descritos. Médicos e enfermeiros recebem na unidade a população das áreas de todos os ACSs de uma mesma equipe, o que também certamente explica uma identificação significativamente maior de diversas situações enumeradas por parte dos profissionais com graduação em saúde.

Outro aspecto importante a ser ressaltado é a convivência de profissionais de saúde em formação com os ACSs, um exercício de interdisciplinaridade que tem sido descrito como benéfico para ambas as partes e, ainda mais, para a população, que se beneficia por uma atenção integral, precoce e resolutiva à saúde (24).

Quando considerada a localização da unidade de saúde do entrevistado (zona urbana ou rural), as situações encontradas com potencialidade para a atuação da fisioterapia parecem ser semelhantes. No entanto, uma porcentagem significativamente maior ( $\mathrm{p}=0,04)$ de profissionais da zona rural (rural: 94,4\% - urbana: 84,7\%) referiu a existência de pessoas com doenças osteomusculares que necessitam atendimento e/ou orientação quanto ao posicionamento, alongamento, analgesia, fortalecimento e retorno às atividades funcionais.

Esse resultado poderia ser explicado pelo próprio predomínio de agravos ocupacionais decorrentes do trabalho físico excessivo de atividades da zona rural, o que justificaria uma maior identificação de doenças osteomusculares na população pelos profissionais dessas áreas (25).

A perspectiva de atuação do profissional fisioterapeuta na qual há maior prevalência de agravos como os indicados em nosso estudo parece ir ao encontro 
da superação das reconhecidas dificuldades das unidades da zona rural. A presença desse tipo de profissional nessas unidades poderia contribuir para a prevenção da instalação de quadros crônicos de limitação, bem como aumentar o grau de conhecimento da população circunscrita sobre formas de promoção de sua saúde e prevenção de agravos à mesma.

Percepção/opinião dos trabalhadores sobre o preparo da equipe atual para atender às situações com potencialidade para atuação da fisioterapia na comunidade

O conjunto de trabalhadores foi questionado sobre sua percepção/opinião quanto ao preparo da equipe em atuação na unidade (capacidade de resolubilidade) para a atenção às situações descritas anteriormente. Foram comparadas as respostas dos que haviam identificado uma determinada situação na sua prática diária com as dos que não a tinham identificado, de forma a verificar se as repostas (equipe preparada ou não preparada) não sofreriam a influência do fato de o trabalhador ainda não haver vivenciado uma determinada situação (Tabelas 4A e 4B).

Os resultados mostram que, na maioria das situações apontadas, não houve diferença entre os dois grupos, exceto quanto ao item orientação de prevenção de quedas em idosos, em que uma porcentagem significativamente maior $(p=0,03)$ de profissionais que não havia identificado a situação em sua prática diária mencionou que a equipe estava preparada para realizar essa atividade $(58,7 \%)$, se comparada aos que já haviam vivenciado essa situação $(37,2 \%)$.

Os dados mostram que a percepção/opinião dos profissionais sobre sua preparação para atender às diferentes situações apresentadas independe do fato de terem ou não já vivenciado a mesma em sua prática diária. A exceção verificada chama a atenção para uma parcela da população crescente no país que, frequentemente, é muito beneficiada com a atuação promotora de saúde exercida pela fisioterapia.

Estudos mostram que fortalecimento muscular adequado e treino de equilíbrio prescrito em casa por profissional de saúde especializado são instrumentos importantes na prevenção de quedas em idosos (26). A necessidade de planejamento de ações para prevenção de quedas em idosos pelas equipes ESF tem sido enfatizada em razão da alta prevalência desses eventos. Estima-se que 30\% da população acima de 65 anos caiam a cada ano (26), sendo que a queda em idosos é acompanhada de sérias consequências físicas, psicológicas e sociais (27).

As tabelas 4A e 4B mostram, ainda, que após a separação dos trabalhadores em função da formação graduada na área da saúde, verificou-se que, em 8 das 14 situações apresentadas, discordaram significativamente ao considerarem a equipe preparada para o enfrentamento das mesmas: atividades físicas e orientações quanto ao autocuidado para diabéticos; atividades físicas e orientações para melhorar a qualidade de vida de hipertensos; assistência e orientação sobre prevenção de escaras, posicionamento, alongamento fortalecimento em pessoas acamadas; assistência e orientação sobre analgesia, posicionamento, alongamento, fortalecimento e retorno às atividades funcionais em pessoas com doenças osteomusculares; treino de marcha e deambulação; higiene brônquica e orientação respiratória para adultos com DPOC; assistência especializada a deficientes físicos; higiene brônquica e orientação respiratória a crianças com doenças pulmonares.

Os profissionais do grupo NGS se dizem mais preparados para atender às diferentes situações enunciadas. Sendo assim, esses dados poderiam expressar uma sobrecarga dos agentes em relação a uma cobrança quanto à resolubilidade da ESF (28). Estudo realizado por Fabricio et al. mostraram que o atendimento às demandas da comunidade representa uma temática emergencial e geradora de vulnerabilidades ao grupo de agentes, pois a eles coube a solução imediata para os problemas na relação comunidade e serviço.

Apesar das diferenças entre os grupos, as tabelas 4A e 4B mostram que, em quatro situações apresentadas, mais da metade dos trabalhadores se sentem despreparados para enfrentá-las: orientação e estímulo a crianças com atraso do desenvolvimento neuropsicomotor (51,7\%), treino de marcha para pessoas com dificuldade para deambular $(51,8 \%)$, assistência especializada e/ou reabilitação para deficientes físicos (68,1\%), assistência especializada e/ou reabilitação para amputados com ou sem próteses $(57,0 \%)$.

O PSF deve dar resolubilidade a $80 \%$ dos problemas de saúde apresentados pela população com assistência contínua e qualificada, melhorando os indicadores de saúde (3).

Nossos achados evidenciam que a equipe de saúde, com sua composição atual, se considera despreparada para a atenção à saúde de uma parte da demanda da população. Com isso, pela ausência de outro(s) 
Tabela 4A - Percepção/opinião dos trabalhadores da ESF, graduados em saúde (GS) e não graduados (NGS), quanto ao preparo da equipe para resolubilidade de situações com potencialidade para atuação da fisioterapia na atenção básica, identificadas (sim) ou (não) em sua prática diária, Rio Grande, RS, 2009

\begin{tabular}{|c|c|c|c|c|c|c|c|}
\hline & \multicolumn{6}{|c|}{ Situação da equipe } & \multirow[b]{3}{*}{ p } \\
\hline & \multicolumn{2}{|c|}{ Preparada } & \multicolumn{2}{|c|}{ Parcialmente preparada } & \multicolumn{2}{|c|}{ Despreparada } & \\
\hline & $\mathrm{n}$ & $\%$ & $\mathrm{n}$ & $\%$ & $\mathrm{n}$ & $\%$ & \\
\hline \multicolumn{8}{|c|}{ Preparo de gestantes para o parto e aleitamento materno } \\
\hline $\operatorname{sim}$ & 48 & 32,4 & 87 & 58,8 & 13 & 8,8 & 0,80 \\
\hline Não & 16 & 34,8 & 25 & 54,3 & 05 & 10,9 & \\
\hline GS & 11 & 22,0 & 33 & 66,0 & 06 & 12,0 & 0,14 \\
\hline NGS & 53 & 36,8 & 79 & 54,9 & 12 & 8,3 & \\
\hline \multicolumn{8}{|c|}{ Atividades físicas e orientações quanto ao autocuidado para diabéticos } \\
\hline Sim & 80 & 45,7 & 91 & 52,0 & 04 & 2,3 & 0,60 \\
\hline Não & 10 & 50,0 & 09 & 45,0 & 01 & 5,0 & \\
\hline GS & 13 & 26,0 & 36 & 72,0 & 01 & 2,0 & $0,03^{*}$ \\
\hline NGS & 77 & 53,1 & 64 & 44,1 & 04 & 2,8 & \\
\hline \multicolumn{8}{|c|}{ Atividades físicas e orientações para melhorar a qualidade de vida de hipertensos } \\
\hline $\operatorname{Sim}$ & 82 & 46,3 & 91 & 51,4 & 04 & 2,3 & 0,10 \\
\hline Não & 12 & 66,7 & 05 & 27,8 & 01 & 5,6 & \\
\hline GS & 12 & 24,0 & 38 & 76,0 & 00 & 0,0 & $0,00^{*}$ \\
\hline NGS & 82 & 56,6 & 58 & 40,0 & 05 & 3,4 & \\
\hline \multicolumn{8}{|c|}{ Orientação e estimulação a cianças com atraso do DNPM* } \\
\hline Sim & 05 & 3,9 & 54 & 42,5 & 68 & 53,5 & 0,80 \\
\hline Não & 03 & 4,8 & 28 & 45,2 & 31 & 50,0 & \\
\hline GS & 01 & 2,0 & 17 & 34,0 & 32 & 64,0 & 0,14 \\
\hline NGS & 07 & 5,0 & 65 & 46,8 & 67 & 48,2 & \\
\hline \multicolumn{8}{|c|}{ Assistência e orientação sobre prevenção de escaras, posicionamento, alongamento e fortalecimento em pessoas acamadas } \\
\hline $\operatorname{Sim}$ & 57 & 34,8 & 100 & 61,0 & 07 & 4,3 & 0,30 \\
\hline Não & 12 & 40,0 & 15 & 50,0 & 03 & 10,0 & \\
\hline GS & 11 & 22,0 & 37 & 74,0 & 02 & 4,0 & $0,04^{*}$ \\
\hline NGS & 58 & 40,3 & 78 & 54,2 & 08 & 5,6 & \\
\hline \multicolumn{8}{|c|}{$\begin{array}{l}\text { Assistência e orientação sobre analgesia, posicionamento, alongamento, fortalecimento } \\
\text { e retorno às atividades funcionais em pessoas com doenças osteomusculares }\end{array}$} \\
\hline Sim & 22 & 12,9 & 112 & 65,5 & 37 & 21,6 & 0,10 \\
\hline Não & 06 & 26,1 & 14 & 60,9 & 03 & 13,0 & \\
\hline GS & 01 & 2,0 & 35 & 70,0 & 14 & 28,0 & $0,01^{*}$ \\
\hline NGS & 27 & 18,6 & 92 & 63,4 & 26 & 17,9 & \\
\hline \multicolumn{8}{|c|}{ Orientação sobre prevenção de agravos crônico-degenerativos e manutenção da saúde } \\
\hline $\operatorname{Sim}$ & 41 & 28,7 & 84 & 58,7 & 18 & 12,6 & 0,90 \\
\hline Não & 13 & 27,1 & 28 & 58,3 & 07 & 14,6 & \\
\hline GS & 10 & 20,0 & 34 & 68,0 & 06 & 12,0 & 0,25 \\
\hline NGS & 44 & 31,2 & 78 & 55,3 & 19 & 13,5 & \\
\hline
\end{tabular}

Fonte: Dados da pesquisa.

Legenda: $\mathrm{n}=$ número de trabalhadores; * $=$ diferença significativa entre os grupos GS e NGS. 
Tabela 4B - Percepção/opinião dos trabalhadores da ESF, graduados em saúde (GS) e não graduados (NGS), quanto ao preparo da equipe para resolubilidade de situações com potencialidade para atuação da fisioterapia na atenção básica, identificadas (sim) ou (não) em sua prática diária, Rio Grande, RS, 2009

\begin{tabular}{|c|c|c|c|c|c|c|c|}
\hline & \multicolumn{6}{|c|}{ Situação da equipe } & \multirow[b]{3}{*}{$\mathrm{p}$} \\
\hline & \multicolumn{2}{|c|}{ Preparada } & \multicolumn{2}{|c|}{ Parcialmente preparada } & \multicolumn{2}{|c|}{ Despreparada } & \\
\hline & $\mathrm{n}$ & $\%$ & $\mathrm{n}$ & $\%$ & $\mathrm{n}$ & $\%$ & \\
\hline \multicolumn{8}{|c|}{ Treino de marcha para pessoas com dificuldade para deambular } \\
\hline $\operatorname{Sim}$ & 18 & 11,1 & 71 & 43,8 & 73 & 45,1 & 0,20 \\
\hline Não & 04 & 13,8 & 08 & 27,6 & 17 & 58,6 & $0,03^{*}$ \\
\hline GS & 01 & 2,0 & 25 & 50,0 & 24 & 48,0 & \\
\hline NGS & 21 & 14,9 & 54 & 38,3 & 66 & 46,8 & \\
\hline \multicolumn{8}{|c|}{ Higienização brônquica e orientação respiratória para adultos com DPOC* } \\
\hline $\operatorname{Sim}$ & 34 & 21,3 & 85 & 53,1 & 41 & 25,6 & 0,70 \\
\hline Não & 08 & 26,7 & 14 & 46,7 & 08 & 26,7 & \\
\hline GS & 04 & 8,0 & 31 & 62,0 & 15 & 30,0 & $0,02^{*}$ \\
\hline NGS & 38 & 27,1 & 68 & 48,6 & 34 & 24,0 & \\
\hline \multicolumn{8}{|c|}{ Higienização brônquica e orientação respiratória para crianças com doenças pulmonares } \\
\hline Sim & 29 & 21,3 & 79 & 58,1 & 28 & 20,6 & 0,30 \\
\hline Não & 15 & 27,3 & 26 & 47,3 & 14 & 25,5 & \\
\hline GS & 05 & 10,0 & 32 & 64,0 & 13 & 26,0 & $0,03^{*}$ \\
\hline NGS & 39 & 27,7 & 73 & 51,8 & 29 & 20,6 & \\
\hline \multicolumn{8}{|c|}{ Assistência especializada e/ou reabilitação para deficientes físicos } \\
\hline $\operatorname{Sim}$ & 13 & 8,1 & 51 & 31,7 & 97 & 60,2 & 0,10 \\
\hline Não & 00 & 0,0 & 07 & 24,1 & 22 & 75,9 & \\
\hline GS & 00 & 0,0 & 12 & 24,0 & 38 & 76,0 & $0,02^{*}$ \\
\hline NGS & 13 & 9,3 & 46 & 32,9 & 81 & 57,9 & \\
\hline \multicolumn{8}{|c|}{ Orientação a idosos quanto à prevenção de quedas } \\
\hline $\mathrm{Sim}$ & 55 & 37,2 & 80 & 54,1 & 13 & 8,8 & $0,03^{\star *}$ \\
\hline Não & 27 & 58,7 & 16 & 34,8 & 03 & 6,5 & \\
\hline GS & 17 & 34,0 & 29 & 58,0 & 04 & 8,0 & 0,33 \\
\hline NGS & 66 & 45,5 & 67 & 46,2 & 12 & 8,3 & \\
\hline \multicolumn{8}{|c|}{ Orientação quanto a postura e autocuidado para cuidadores de pessoas acamadas } \\
\hline Sim & 52 & 33,8 & 85 & 55,2 & 17 & 11,0 & 0,80 \\
\hline Não & 13 & 31,7 & 22 & 53,7 & 06 & 14,6 & \\
\hline GS & 12 & 24,0 & 29 & 58,0 & 09 & 18,0 & 0,13 \\
\hline NGS & 53 & 36,6 & 78 & 53,8 & 14 & 9,7 & \\
\hline \multicolumn{8}{|c|}{ Assistência especializada e/ou reabilitação para amputados com ou sem próteses } \\
\hline Sim & 08 & 6,5 & 45 & 36,6 & 70 & 56,9 & 0,40 \\
\hline Não & 08 & 11,4 & 22 & 31,4 & 40 & 57,1 & \\
\hline GS & 01 & 2,0 & 15 & 30,6 & 33 & 67,3 & 0,09 \\
\hline NGS & 15 & 10,4 & 52 & 36,1 & 77 & 53,5 & \\
\hline
\end{tabular}

Fonte: Dados da pesquisa.

Legenda: $\mathrm{n}=$ número de trabalhadores; * $=$ diferença significativa entre os grupos GS e NGS. 
profissional(is) na equipe, essa parte da população com necessidades, que poderia ser atendidas na própria comunidade (unidade e/ou domicílio), acaba sendo referenciada para procedimentos de maior complexidade. No caso específico da fisioterapia, alguns autores têm descrito que as condições socioeconômicas desfavoráveis e a impossibilidade de transporte são fatores de repressão da demanda de atendimento pela fisioterapia (29).

Há consequências que poderiam ser evitadas, como agravamento de determinadas situações, deslocamentos desnecessários da população, retardo na resolubilidade das situações e ônus ao sistema, tanto para a população quanto para os gestores em saúde.

Além disso, é importante observar que quando são analisadas em conjunto as respostas parcialmente preparada e despreparada, a condição de preparo inadequado da equipe estende-se para a maioria das situações investigadas (Tabelas 4Ae 4B).

Uma capacitação inadequada tem sido apontada como um dos fatores que poderiam explicar esses resultados. Em um estudo sobre a organização do trabalho e perfil dos profissionais do PSF em um município de Minas Gerais, Cotta (30) aponta a questão da qualificação profissional dos trabalhadores da ESF como obstáculo real para uma implantação mais adequada dessa estratégia segundo seus princípios norteadores, que têm o coletivo e a família como focos de atenção.

Ainda dentro da perspectiva da capacitação para ação, a necessidade de uma formação mais adequada dos novos profissionais de saúde tem sido identificada como um alvo a ser seguido para melhorar a resolubilidade da ESF. Nesse sentido, algumas iniciativas dirigidas aos estudantes de graduação, como o PRÓSAÚDE (31), e a implantação de cursos em nível de pós-graduação (especialização e residência em saúde da família) constituem-se em esforços do Ministério da Saúde para adequar os profissionais ao novo perfil de profissional desejado para atuar na ESF. Esse profissional deve estar capacitado para planejar, organizar, desenvolver e avaliar ações que respondem às reais necessidades da comunidade, articulando os diversos setores envolvidos na Promoção de Saúde (31).

Também não se pode deixar de ponderar que a própria constituição das equipes, como se encontram hoje, pode ser um fator limitante para a resolubilidade das situações encontradas pelos trabalhadores na sua prática diária. Esse problema poderia ser equacionado com a inclusão de outros profissionais na equipe, entre eles, o fisioterapeuta. 0 potencial para a promoção da saúde da fisioterapia e a própria atenção fisioterapêutica, nas diversas situações identificadas na comunidade pelos profissionais de saúde, poderiam contribuir de forma significativa para o aumento da efetividade da ESF no município, gerando menos gastos para o sistema de saúde como um todo.

Quando os trabalhadores foram dicotomizados quanto à localização da unidade de saúde, as respostas foram significativamente diferentes $(p=0,01)$ quanto ao item assistência especializada e/ou reabilitação para amputados com ou sem próteses: equipe preparada (urbana 5,6\%; rural 13,0\%), parcialmente preparada (urbana 41,1\%; rural 23,2\%), despreparada (urbana 53,2\%; rural 63,8 \%).

A eficácia da ESF na zona rural tem sido estudada por alguns autores. Fatores como a distância dos centros urbanos, o tamanho das circunscrições rurais e a dispersão da população são descritos por Medina e Hartz (32) como limitadores da resolubilidade da ESF em zonas rurais. A distância das unidades em relação à Secretaria Municipal de Saúde, dificuldades operacionais de transporte e falta de pessoal capacitado foram descritos (16) como grandes desafios para a atenção básica na região sul do país.

Apesar da diferença significativa entre os grupos, nota-se que tanto na zona urbana como na zona rural, a maioria dos trabalhadores refere o despreparo da equipe para a atenção a indivíduos amputados. Mais uma vez, defronta-se com uma situação com a qual a equipe se sente despreparada para a atenção integral. A atenção à saúde de amputados não só pelo trauma vivenciado pelo paciente e seus familiares, mas pelas consequências multidimensionais que podem advir dessa condição, representa um desafio para os profissionais de saúde e, em especial, para a fisioterapia (33). 0 número de amputações no país e, particularmente, em nossa região é uma realidade crescente, sobretudo em virtude do aumento de acidentes de trânsito, com destaque para aqueles com envolvimento de motociclistas (34).

Infraestrutura da unidade de saúde na perspectiva da atuação do fisioterapeuta

No que se refere à estrutura física das unidades de saúde, verificou-se que o bom estado geral do prédio foi indicado pela maioria dos trabalhadores em 12 das 19 unidades investigadas $(p=0,00)$, sendo que, em quatro unidades $(21,1 \%)$, a boa avaliação foi unânime. 
Considerando-se as especificidades de uma parte da clientela potencialmente beneficiada, foram investigados ainda outros itens de infraestrutura que seriam necessários para a atuação do fisioterapeuta na unidade de saúde. Os itens investigados e a porcentagem de unidades que apresentavam os mesmos foram: rampa de acesso (36,8\%); bancos de espera para o atendimento $(100,0 \%)$; banheiros adaptados a portadores de necessidades especiais $(26,3 \%)$ e salas de atendimento de outras especialidades $(31,6 \%)$.

A facilidade de acesso à unidade foi indicada por mais de 50\% dos trabalhadores em 13 unidades, sendo que, em 5 delas (26,3\%), houve unanimidade em relação a esse item. Nesse sentido, a garantia do acesso possibilita que a ESF seja a porta de entrada para o SUS, facilitando o estabelecimento do vínculo entre a equipe e o usuário.

Avaliando as estruturas físicas das Unidades de Saúde da Família da região sul, Facchini et al. (16) destacaram a precariedade e a improvisação das mesmas, salientando que um maior investimento na adaptação das unidades para atividades que não estão centradas no médico e no usuário individual poderá contribuir para o melhor desempenho do modelo de atenção básica e da gestão dos serviços de saúde. Para esses autores, os investimentos em infraestrutura para reformas e construção de UBS na concepção do ESF são urgentes em virtude dos requisitos legais de conforto e segurança ambiental.

Nossos resultados mostram que, embora a maioria das unidades não se encontre adaptada para o atendimento na unidade por outras especialidades, 6 das 19 unidades atuais já apresentam condições físicas mínimas necessárias para o exercício da fisioterapia em nível ambulatorial.

Conhecimento sobre os níveis de atuação e autonomia da profissão e aproximação com a Fisioterapia

A maioria dos trabalhadores $(87,2 \%)$ indicou ser uma profissão que promove a saúde, previne agravos à mesma e reabilita sequelas funcionais. Dados semelhantes foram encontrados em um estudo sobre os conhecimentos e as atitudes da equipe do PSF em relação à Fisioterapia no município de Londrina, PR (13).

Quanto ao entendimento dos entrevistados sobre a formação em Fisioterapia e a autonomia da profissão, a maioria dos entrevistados $(69,2 \%)$ mencionou que se trata de um curso superior com autonomia para definir condutas próprias. Porém, apesar do bom conhecimento dos sujeitos da pesquisa em relação à Fisioterapia, o contato na equipe de trabalho foi citado por apenas $15,3 \%$ da totalidade de trabalhadores da ESF do município.

$\mathrm{Na}$ análise categorizada em graduados e não graduados em saúde, verificou-se que $62 \%$ dos trabalhadores do grupo GS e $91,7 \%$ do grupo GNS nunca tiveram contato com a Fisioterapia em suas equipes de trabalho, com diferença significativa entre os grupos $(\mathrm{p}=0,00)$. Entre os trabalhadores que tiveram contato prévio com a Fisioterapia, este se deu em hospitais $(10,2 \%)$ e/ou clínicas de reabilitação $(2,0 \%)$.

Estudo realizado por Aguiar (12) mostrou que o grau de conhecimento da equipe se mostrou suficiente para que os profissionais que atuam junto à comunidade no PSF saibam identificar em quais casos há a possibilidade de intervenção fisioterapêutica. Nossos dados indicam que, apesar do bom conhecimento teórico indicado pelos entrevistados sobre a área de atuação da Fisioterapia, a identificação de situações nas quais ela pode aumentar a resolubilidade da estratégia pode estar prejudicada em razão do pouco contato profissional com essa área em outros cenários de atuação além da prática curativa e reabilitadora anteriormente descrita.

Em estudo de prevalência de utilização da fisioterapia pela população de Pelotas (RS), apenas 4,9\% da amostra haviam recebido serviços dessa área no anterior à pesquisa (23). Um dado importante é que $94,9 \%$ dos atendimentos foram a patologias já instaladas e tratamentos pós-cirúrgicos. Esses dados, somados aos nossos resultados, evidenciam a grande lacuna deixada pela Fisioterapia não só em relação ao espaço na equipe multidisciplinar, mas também perante a população na promoção de saúde e nas ações de produção de saúde.

\section{Conclusão}

Nosso estudo evidencia a necessidade da inserção da Fisioterapia na ESF como contribuição efetiva para a resolubilidade da estratégia e para a consolidação dos princípios norteadores do SUS. Porém, essa inserção deve ser subsidiada por informações de demandas da população e infraestrutura local, de forma a assegurar a eficácia da ação. 
Uma limitação da pesquisa se deve ao número reduzido de situações descritas aos trabalhadores cuja atuação da Fisioterapia já é reconhecida. Sabe-se de outras contribuições da profissão no âmbito da atenção básica à saúde, porém a fundamentação teórica para a elaboração de um instrumento torna-se restrita, uma vez que as publicações nessa área são em pequeno número.

Ressalta-se que o profissional que venha compor a equipe deve realizar uma interação e divulgação do campo de trabalho junto aos demais profissionais (médicos, enfermeiros, técnico e auxiliares de enfermagem e ACSs). Além disso, visitas domiciliares junto aos ACSs são necessárias, em função das diferenças de percepção de tais profissionais em relação à identificação das situações descritas.

São enumeradas as situações em que há a identificação da demanda pela equipe e, ao mesmo tempo, a indicação do preparo para o enfrentamento de tais situações, com consequente impacto à resolubilidade da estratégia.

As diferenças de percepção entre os graduados e não graduados em saúde devem ser consideradas e abordadas pelo profissional inserido à equipe, sob a luz da interdisciplinaridade. Além disso, devem ser planejadas e executadas ações que levem em consideração as diferenças apontadas com relação à localização da unidade (zona rural e urbana).

Espera-se, com este trabalho, contribuir para a apropriação da Fisioterapia no campo da Promoção de Saúde no âmbito da Saúde da Família, bem como de outras profissões que vislumbram as mesmas possibilidades, com o objetivo de contribuir para a saúde integral da população.

\section{Referências}

1. Sampaio RF. Promoção de saúde, prevenção de doenças e incapacidades: a experiência da fisioterapia/ UFMG em uma unidade básica de saúde. Fisioter Mov. 2002;15(1):19-23.

2. Saldanha CRM. Avaliação do PSF em Juiz de Fora. [acesso 8 fev. 2010]. Disponível em: http://www.opas. org.br/servico/arquivos/Sala5325.pdf.

3. Brasil. Ministério da Saúde. Secretaria de Vigilância em Saúde. Política nacional de promoção da saúde/ Ministério da Saúde, Secretaria de Atenção à Saúde. Brasília: Ministério da Saúde; 2006.
4. Silva D, da Ros MA. Inserção de profissionais de fisioterapia na equipe de saúde da família e Sistema Único de Saúde: desafios na formação. Ciênc Saúde Coletiva. 2007;12(6):1673-81. doi.org/10.1590/S141381232007000600028 .

5. Pinheiro R, Ceccim RB, de Mattos RA. Ensinar saúde: a integralidade e o SUS nos cursos de graduação da área de saúde. Rio de Janeiro: IMS/UERJ;ABRASCO; 2005.

6. Ferreira VA, Magalhães R. Nutrição e promoção da saúde: perspectivas atuais. Cad. Saúde Pública. 2007; 23(7):1674-81.

7. Oliveira EM, Spiri WC. PSF: experiência da equipe multiprofissional. Rev Saúde Pública. 2006;40(4): 727-33.

8. Barros FBM. Autonomia profissional do fisioterapeuta ao longo da história. Rev FisioBrasil. 2003;(59):20-31.

9. Ribeiro KSQS. A experiência na extensão popular e a formação acadêmica em fisioterapia. Cad. CEDES. 2009;29(79):335-46.

10. Saito RXS, Lacerda RA, Fracolli LA. Atenção primária: análise de acesso ao primeiro contato em uma Unidade Básica de Saúde do município de São Paulo. Rev Paul Enferm. 2006;25(2):74-81.

11. Ragasson CA. Atribuições do fisioterapeuta no programa de saúde da família. Reflexões a partir da prática profissional. [acesso 20 ago. 2008]. Disponível em: http://www.crefito5.com.br/web/downs/ psf_ado_fisio.pdf.

12. Aguiar RG. Conhecimentos e atitudes sobre atuação profissional do fisioterapeuta entre os profissionais da equipe mínima de saúde da família em Ribeirão Preto. [dissertação]. Faculdade de Medicina de Ribeirão Preto-USP; São Paulo; 2005.

13. Trelha CS, Silva DW, Iida LM, Fortes MH, Mende TS. O fisioterapeuta no programa de saúde da família em Londrina (PR). Rev Esp Saúde. 2007;8(2):20-5.

14. de Rezende M, Rasga MM, Amâncio A Filho, Tavares MFL. A equipe multiprofissional da "Saúde da Família": uma reflexão sobre o papel do fisioterapeuta. Ciênc saúde coletiva. 2009;14(Supl. 1):1403-10.

15. Chartered Society OF Physiotherapy. Physiotherapy effectiveness bulletin - health promotion: physical activity and exercise in selected populations. Canada. 2002;4(1):2-11. 
16. Facchini LA, Piccini RX, Tomasi E, Thumé E, Silveira DS, Siqueira FV, et al. Desempenho do PSF no Sul e no Nordeste do Brasil: avaliação institucional e epidemiológica da Atenção Básica à Atenção Básica à Saúde. Ciênc Saúde Coletiva. 2006;11(3):669-81.

17. Brasil. Ministério da Saúde. Cad Informações de Saúde. 2009. [acesso nov. 2009]. Disponível em: http:// tabnet.datasus.gov.br/tabdata/cadernos/RS/RS_Rio_ Grande_Geral.xls.

18. Loch-Neckel G, Giane S, Berton EH, Moroni RM, Crepaldi MA. Desafios para a ação interdisciplinar na atenção básica: implicações relativas à composição das equipes de saúde da família. Ciênc Saúde Coletiva. 2009; 14(Supl. 1):1463-72.

19. Ministério da Saúde. Kell MCG. [Adapt.] Integralidade da atenção à saúde. Secretaria de Assistência à Saúde. Secretaria de Atenção à Saúde. Departamento de Atenção Básica. Coordenação de Gestão da Atenção Básica. [acesso 5 dez. 2008]. Disponível em: http:// www.opas.org.br/observatorio/Arquivos/Destaque 69.doc.

20. Mattos R. Integralidade como eixo da formação dos profissionais de saúde. Rev Bras Educ Méd. 2004; 28(2):91-2.

21. Brasil. Ministério da Saúde. Portaria GM 154. Diário Oficial da República Federativa do Brasil, Poder Legislativo, 25 jan. 2008, n. 18, Seção 1, p. 47 a 49.

22. Almeida AB, Oliveira AMB, Ribeiro KSQS. A fisioterapia na atenção básica a partir de uma experiência de educação popular. In: Anais do V Colóquio Internacional Paulo Freire, Recife, 2005.

23. Siqueira FV, Facchini LA, Hallal PC. Epidemiologia da utilização de fisioterapia em adultos e idosos. Rev Saúde Pública. 2005;39(4):663-8.

24. Pedrosa JIS, Teles JBM. Consenso e diferenças em equipes do Programa Saúde da Família. Rev Saúde Pública. 2001;35(3):303-11.

25. Buchabqui JÁ, Capp E, Petuco DRS. Convivendo com agentes de transformação: a interdisciplinaridade no processo de ensino/aprendizado em saúde. Rev Bras Educ Méd. 2006;30(1):32-8. doi.org/10.1590/S010055022006000100006 .

26. Wünsch V Filho. Perfil epidemiológico dos trabalhadores. Rev Bras Med Trab. 2004;2(2):103-17.
27. Gillespie LD, Gillespie WJ, Robertson MC, Lamb SE, Cumming RG, Rowe BH. Interventions for preventing falls in elderly people. Cochrane Database Syst Rev. 2003;(4):CD000340.

28. Fabricio SCC, Rodrigues RAP, Costa ML Jr. Falls among older adults seen at a São Paulo State public hospital: causes and consequences. Rev. Saúde Pública. 2004; 38(1):93-9.

29. Arruda AD, Guedes BN, Lima FR, Ribeiro KSQS, Cavalcanti RLL. A importância da inclusão da fisioterapia no programa saúde da família. Universidade Federal da Paraíba. [acesso 2 maio 2010]. Disponível em: http:// www.prac.ufpb.br/anais/anais/saude/fisioterapia. pdf.

30. Cotta RMM, Schott M, Azeredo CM, Franceschini SCC, Priore SE, Dias G. Organização do trabalho e perfil dos profissionais do programa saúde da família: um desafio na reestruturação da atenção básica em saúde. Epidemiol Serv Saúde. 2006;15(3):7-18.

31. Brasil. Ministério da Saúde. Secretaria de Gestão do Trabalho e da Educação na Saúde. Programa Nacional de Reorientação da Formação Profissional em Saúde (Pró - Saúde). [acesso 20 mar. 2010]. Disponível em: http://prosaude.org/not/prosaude-maio2009/proSaude.pdf.

32. Medina MG, Hartz ZMA. The role of the family health program in the organization of primary care in municipal health systems. Cad Saúde Pública. 2009;25(5): 1153-67.

33. Barauna MA, Duarte F, Sanchez HM, Canto RST, Malusá S, Campelo-Silva CD, et al. Avaliação do equilíbrio estático em indivíduos amputados de membros inferiores através da biofotogrametria computadorizada. Rev Bras Fisioter. 2006;10(1):83-90.

34. Calil AM, Sallum EA, Domingues CA, Nogueira LS. Mapping injuries in traffic accident victims: a literature review. Rev Lat Am Enfermagem. 2009;17(1):120-5.

Recebido: 03/04/2011 Received: 04/03/2011

Aprovado: 02/08/2011 Approved: 08/02/2011 